\title{
Phylogenomics picks out the par excellence markers for species phylogeny in the genus Staphylococcus.
}

\author{
Lucia Graña-Miraglia ${ }^{1}$, César Arreguín-Pérez ${ }^{2}$, Gamaliel Lopez-Leal ${ }^{3}$, Alan Muñoz ${ }^{1}$, Angeles Pérez-Oseguera

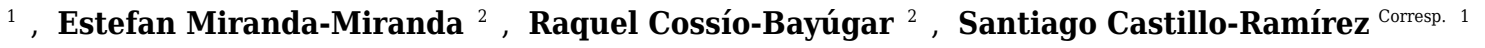 \\ 1 Programa de Genómica Evolutiva, Centro de Ciencias Genómicas, Universidad Nacional Autónoma de México, Cuernavaca, Morelos, Mexico \\ 2 Centro Nacional de Investigación Disciplinaria en Parasitología Veterinaria del Instituto Nacional de Investigaciones Forestales, Agrícolas y Pecuarias, \\ Jiutepec, Morelos, Mexico \\ 3 Departamento de Microbiología Molecular, Instituto de Biotecnología, Universidad Nacional Autónoma de México, Cuernavaca, Morelos, Mexico \\ Corresponding Author: Santiago Castillo-Ramírez \\ Email address: iago@ccg.unam.mx
}

Although genome sequencing has become a very promising approach to conduct microbial taxonomy, few labs have the resources to afford this especially when dealing with data sets of hundreds to thousands of isolates. The goal of this study was to identify the most adequate loci for inferring the phylogeny of the species within the genus Staphylococcus; with the idea that those who cannot afford whole genome sequencing can use these loci to carry out species assignation confidently. We retrieved 177 orthologous groups (OGs) by using a genome-based phylogeny and an average nucleotide identity analysis. The top 26 OGs showed topologies similar to the species tree and the concatenation of them yielded a topology almost identical to that of the species tree. Furthermore, a phylogeny of just the top 7 OGs could be used for species assignment. We sequenced four staphylococcus isolates to test the $26 \mathrm{OGs}$ and found that these OGs were far superior to commonly used markers for this genus. On the whole, our procedure allowed identification of the most adequate markers for inferring the phylogeny within the genus Staphylococcus. We anticipate that this approach will be employed for the identification of the most suitable markers for other bacterial genera and can be very helpful to sort out poorly classified genera. 
1 Phylogenomics picks out the par excellence markers for species phylogeny in the genus

2 Staphylococcus.

3

4

5 Authors

6 Lucía Graña-Miraglia ${ }^{1}$, César Arreguín-Pérez ${ }^{2}$, Gamaliel López-Leal ${ }^{3}$, Alan Muñoz ${ }^{1}$, Ángeles Pérez-

7 Oseguera ${ }^{1}$, Estefan Miranda-Miranda ${ }^{2}$, Raquel Cossío-Bayúgar ${ }^{2}$ and Santiago Castillo-Ramírez ${ }^{1 *}$.

8

9 1Programa de Genómica Evolutiva, Centro de Ciencias Génomicas, Universidad Nacional Autónoma de 10 México, Cuernavaca, Morelos 62210, México.

$11{ }^{2}$ Centro Nacional de Investigación Disciplinaria en Parasitología Veterinaria del Instituto Nacional de

12 Investigaciones Forestales, Agrícolas y Pecuarias, Carr. Fed. Cuernavaca-Cuautla No. 8534, Jiutepec,

13 Morelos, 62550 México.

$14{ }^{3}$ Departamento de Microbiología Molecular, Instituto de Biotecnología, Universidad Nacional Autónoma 15 de México, Cuernavaca, México.

16

17

18

*Correspondence: Santiago Castillo-Ramírez, iago@,ccg.unam.mx

19

20

21

22

23

24

25 


\section{ABSTRACT}

27 Although genome sequencing has become a very promising approach to conduct microbial taxonomy,

28 few labs have the resources to afford this especially when dealing with data sets of hundreds to thousands

29 of isolates. The goal of this study was to identify the most adequate loci for inferring the phylogeny of

30 the species within the genus Staphylococcus; with the idea that those who cannot afford whole genome

31 sequencing can use these loci to carry out species assignation confidently. We retrieved 177 orthologous

32 groups (OGs) by using a genome-based phylogeny and an average nucleotide identity analysis. The top

3326 OGs showed topologies similar to the species tree and the concatenation of them yielded a topology

34 almost identical to that of the species tree. Furthermore, a phylogeny of just the top 7 OGs could be used

35 for species assignment. We sequenced four staphylococcus isolates to test the 26 OGs and found that

36 these OGs were far superior to commonly used markers for this genus. On the whole, our procedure

37 allowed identification of the most adequate markers for inferring the phylogeny within the genus

38 Staphylococcus. We anticipate that this approach will be employed for the identification of the most

39 suitable markers for other bacterial genera and can be very helpful to sort out poorly classified genera.

\section{INTRODUCTION}

43 The study of ecology and evolution has been substantially transformed by the omics technologies.

44 Remarkably, the use of these technologies has allowed essential questions in evolutionary biology to be 45 addressed and has advanced our knowledge in many biological processes (López-Leal, Tabche et al. 46 2014, Joseph, Marti et al. 2015, Joseph, Cox et al. 2016). The precise identification of the different 47 species within any given genus is highly valuable for many branches of microbiology. For instance, as far 48 as clinical microbiology is concerned, this is instrumental in establishing which species are human/animal 49 pathogens and which are just regular commensal organisms; whereas in microbial ecology species 50 assignation is very helpful in defining the niche-range of the different species (Becker, Margos et al. 51 2016). 
53 Although whole-genome sequencing is the best method for genotyping bacterial isolates and, therefore, to

54 conduct species assignation/identification, this approach is not yet affordable for routine use in many

55 laboratories all over the world. This is especially true for low and middle-income countries in which the

56 amount of money invested in science is not as much as in developed countries. For this particular

57 purpose, and from a theoretical point of view, orthologous genes are the perfect candidates for inferring

58 the phylogeny of species, as they should reflect the species tree of the taxa considered. As per definition,

59 orthologous genes are the ideal candidates to track the sequence of past of speciation events within a

60 given lineage (Fitch 2000). Therefore, in order to infer the phylogeny of the species, a clear-cut

61 ascertainment of orthologous genes is of paramount importance and phylogenomic pipelines can be

62 implemented to try to identify the potential orthologous genes. Orthologous relationships are context-

63 sensitive and a gene family could be mainly composed of orthologous genes at one taxonomic level but if

64 one considers a higher taxonomic level many more non-orthologous genes will appear. Furthermore,

65 orthologous relationships could involve one to many relationships (Gabaldón and Koonin 2013). The

66 word co-orthologue was coined to describe that exact situation in which a genome has more than one

67 orthologous gene (Gabaldón and Koonin 2013). Hence, from a practical point of view (i.e. operational

68 implementation), orthologous genes with just one gene per genome among the taxa considered should be

69 the best markers to delineate the history of the species.

71 The genus Staphylococcus has a few dozens of species of Gram-positive bacteria, which are commensals

72 colonizing the skin and mucous membranes of some mammals and birds. However, some of these species

73 have a clear clinical and economic relevance, as they are a frequent cause of infection in humans,

74 livestock, and domestic animals. Although the genus is very well known for the human opportunist

75 pathogen S. aureus, which is famous worldwide as major source of nosocomial infections (Challagundla,

76 Reyes et al. 2018, Frisch, Castillo-Ramirez et al. 2018), there are some other species such as $S$.

77 epidermidis, S. lugdunesis, $S$. saprophyticus and $S$. schleiferi that have been associated with human 
78 infections. Several molecular-based methods have been introduced to carry out species identification

79 within the genus Staphylococcus (Kwok and Chow 2003, Ghebremedhin, Layer et al. 2008, Sasaki,

80 Tsubakishita et al. 2010, Lamers, Muthukrishnan et al. 2012). PCR along with sequence analysis of the

81 genes dnaJ, tuf, sodA, rpoB, hsp60 and nuc have been used to differentiate Staphylococcus species (Kwok

82 and Chow 2003, Ghebremedhin, Layer et al. 2008, Sasaki, Tsubakishita et al. 2010, Lamers,

83 Muthukrishnan et al. 2012). Although they have been very useful and demonstrate a better resolution

84 than the 16S rRNA gene (Ghebremedhin, Layer et al. 2008), these genes show different amounts of

85 genetic diversity and, therefore, varying levels of discriminatory power for the different species. Thus,

86 depending on the gene and the species, low-level resolution or even mis-identification can occur. Clearly,

87 an ideal solution would be to conduct whole-genome sequencing of all the isolates considered to tell apart

88 the different species. In terms of genome sequences, Staphylococcus is one of the few bacterial genera for

89 which many genomes are available.

90

91 In this study, we address the question of which genes are the best inter-species markers (bona fide

92 orthologous genes) for the genus Staphylococcus and, by applying a phylogenomic approach, we provide

93 a list of the top candidates for species assignation within this genus. Underfunded groups could use these

94 top candidates to accurately conduct species assignation without the necessity of using whole-genome

95 sequencing. Furthermore, this approach could be used for many other species/genera to identify the most

96 suitable markers for inferring the phylogeny of the taxa under investigation.

\section{METHODS}

\section{Genomes and homologous groups}

100 We downloaded 265 publically available complete genomes (see Supplementary Table 1). These cover 46

101 different species from the genus Staphylococcus and are a good representation of the host range within

102 this genus. We ran CheckM (Parks, Imelfort et al. 2015) on these genomes to discard poorly sequenced

103 genomes (i.e. with contamination or incomplete) and only one of them (marked in red in Supplementary 
104 Table 1 ) did not pass the criteria ( $\geq 95 \%$ complete and with $\leq 5 \%$ contamination) and thus were not

105 included in the rest of the analyses. We also sequenced the genomes of 4 bacterial isolates (see

106 Supplementary Table 2) obtained from hemolymph or hypostome exudates from adult ticks showing

107 signs of bacterial infection and reared over experimentally infested bovines in the Centro Nacional de

108 Investigación Disciplinaria en Parasitología Veterinaria (CENID-PAVET-INIFAP), Jiutepec, Morelos,

109 México. Of note the 4 isolates sequenced were confirmed to be Staphylococcus spp by biochemical tests

110 and 16S rRNA gene sequence analysis. The isolate INIFAP 005-08 was initially identified as $S$.

111 saprophyticus by its physical and biochemical characteristics (Gram-positive coccus bacteria, catalase

112 positive; novobiocin resistant, absence of coagulase, gelatinase and caseinase activity). This isolate was

113 also able to produce acids by fermenting glycerol, lactose, $\mathrm{D}(+)$ mannose, sucrose and turanose; however

114 it was unable to use $\mathrm{L}(+)$ arabinose or $\mathrm{L}(+)$ lactose. This isolates was classified as $S$. xylosus as per 16S

115 rRNA gene analysis. The isolates INIFAP 002-16 and INIFAP 004-15 were identified as Gram-positive

116 coccus bacteria, catalase positive and ribotyped by the $16 \mathrm{~S} r R N A$ gene, which exhibited positive identity

117 for S. xylosus. INIFAP 009-16 was identified as Gram-positive coccus bacteria, catalase-positive and

118 identified as $S$. succinus using the 16S $r R N A$ gene. This same isolate was identified as $S$. carnosus using

119 the API20E (bioMérieux 2010) system. The Nextera XT DNA Library Prep Kit was used for the

120 sequencing libraries and Agilent High Sensitivity DNA Kit was employed for quality control. The isolates

121 were sequenced using an Illumina MiSeq platform, with a 2 X250 bp configuration; the genome

122 sequencing was conducted at Instituto Nacional de Medicina Genómica (http://www.inmegen.gob.mx) in

123 Mexico City. Prior to assembling the genomes, we employed the SolexQA v.3.7.1 program (Cox,

124 Peterson et al. 2010) to trim the reads, employing the default parameters. We used Velvet version 1.2.09

125 (Zerbino and Birney 2008) and Spades v3.11.0 (Bankevich, Nurk et al. 2012) to carry out de novo

126 assembly setting the option careful and we tested the following k-mer sizes:

$12751,61,71,81,91,101,111,121,127$. We did not consider contigs smaller than $300 \mathrm{bp}$ in the final

128 assemblies. In all cases, Spades outperformed Velvet in terms of the number of contigs and the N50

129 statistics. Again, we used CheckM (Parks, Imelfort et al. 2015) to evaluate the quality of the genomes 
130 sequenced by us; notably all the 4 isolates were shown to be $\geq 95 \%$ complete and with $\leq 5 \%$

131 contamination and therefore reliable for downstream analyses. The details of the genomes assemblies are

132 provided in Supplementary Table 2. These seem to be good genome assemblies as judged by the high

133 median coverage and the low number of contigs for each one of the newly sequenced isolates. These 4

134 genomes have been submitted to the GenBank and have the following accession numbers:

135 PIZQ01000000, PIZN00000000, PIZP00000000, PIZO00000000 (BioProject PRJNA421192). Then, we

136 employed PROKKA v1.11 (we set the genus option to Staphylococcus) to annotate all the genomes

137 sequences (newly sequenced ones and the publically available) to have a consistent annotation for all of

138 them. To get the orthologous groups (OGs) with one-to-one relationships among the species, we only

139 considered single gene families (SGF); these were constructed running BLASTP searches with an e-value

140 of $1.0 \mathrm{e}^{-30}$ between the genome of $S$. aureus TW20 and the rest of the genomes. We kept all the cases

141 where there was only one hit per genome and requiring that the seed from TW20 and the hit aligned $\geq 60$

$142 \%$ of their lengths and were $\geq 45 \%$ identical - the rationale behind these two criteria was to make sure

143 that we had whole genes and not just domains. Then, for the all the SGF we constructed DNA alignments

144 in frame via the program Fast statistical alignment version 1.5.9 (Bradley, Roberts et al. 2009), setting the

145 option --nucprot that align nucleotide sequences taking into account the protein space. We conducted

146 recombination analysis on each of the SGFs using PhiTest (Bruen, Philippe et al. 2006) that was

147 implemented via the PhiPack program, using a window size of $50 \mathrm{bp}$. Additionally, we also determined

148 the nucleotide diversity for each one of the SGFs using R pegas library function nuc.div() with the default

149 parameters. We carried out a function enrichment analysis as follows: first, we conducted a Gene

150 Onthology (GO) annotation via InterProScan version 5 using the genes from the S. aureus TW20 genome

151 as a reference. Then, we conducted a GO enrichment analysis via Blast2GO PRO using a Fisher's Exact

152 Test (https://www.blast2go.com/), the analysis was conducted at three different GO levels: Molecular

153 Function (MF), Biological process (BP) and Cellular Component (CC). Then to evaluate the results, we

154 used a False Discovery Rate of 0.05 and the Benjamini-Hochberg correction was used to account for

155 multiple testing. 
157 Phylogenetic reconstructions, average nucleotide identity analysis and neighbour nets.

158 We performed phylogenetic reconstructions for every SGF that did not show recombination signals as per 159 PhiTest (Bruen, Philippe et al. 2006) and for the super alignment (read below). All the gene trees were 160 constructed with RaxML version 8.2.11 (Stamatakis 2014) executing 10 inferences on the alignment, 161 using 10 distinct randomized Maximum Parsimony (MP) trees and with the GTR $+\mathrm{G}+\mathrm{I}$ model. Both the 162 Shimodaira-Hasegawa topology test and the Robinson-Foulds distance were also conducted via RAxML 163 with the default settings. It is known that species tree estimation is not a trivial matter and we employed a 164 previous approach that gave trustable results (Castillo-Ramirez and Gonzalez 2008). We created a super 165 alignment concatenating all the SGFs that did not have signals for recombination and on this alignment a 166 ML phylogeny was constructed also through RAxML, this time executing 20 independent inferences 167 starting from 20 different MP trees and with GTR $+\mathrm{G}+\mathrm{I}$ model. For this ML phylogeny bootstrap 168 replicates were generated again employing RaxML, using the -x option that implements a fast algorithm 169 for bootstrapping. The average nucleotide identity analysis (ANI) was run via the python module pyani 170 (http://widdowquinn.github.io/pyani/), specifically we employed the ANIm method (Richter and 171 Rosselló-Móra 2009). To determine the GC content and the proportion of variable sites for each SGF, we 172 used the function summary from the program AMAS (Borowiec 2016). In order to visualize the 173 conflicting phylogenetic signals in the genes rpoB and tuf we used SplitTree4 (Huson and Bryant 2005) to 174 construct Neighbour nets with uncorrected P distances.

175

176 RESULTS

\section{Defining the species tree, confirming genome affiliations and the set of orthologous genes}

178 We focused on the genus Staphylococcus as it has clear clinical and veterinary relevance and, due to that, 179 it has been extensively covered in terms of genome sequences. The data set employed for this study 180 represents 46 species, incorporating 40 type strains, and included a total of 269 genomes for the analyses

181 (see Supplementary Tables 1 and 2). Notably, this data set has species with a wide host-range covering 
182 many of the niches described for this genus. First, we determined the single gene families (SGFs), as

183 these are potential candidates to be orthologous genes with one-to-one relationships, and found 208 SGFs.

184 However recombination could have affected them and, therefore, we conducted recombination tests on

185 them and around $15 \%$ of them (31 SGFs) showed signals of recombination and were discarded, which left

186177 SGFs as good candidates to be orthologous groups (OG); the list of these 177 SGFs is provided in

187 Supplementary Table 3. We employed a previously used strategy (Castillo-Ramirez and Gonzalez 2008)

188 to approximate the species tree. This is the total evidence approach, in which all the 177 SGFs without

189 signals of recombination are concatenated and treated as if they were a single marker on which a

190 Maximum Likelihood (ML) phylogeny was constructed (see Figure 1) - this ML phylogeny was our

191 Proxy for the Species Tree Topology (PSTT). From Figure 1 one can see that type strains are scattered

192 throughout the tree and that most of the isolates from individual species tend to form monophyletic

193 groups and these groups are very well-supported as most of them have bootstrap values higher than 80

194 (see orange dots in the phylogeny). However, we also noted a region on the tree (shaded areas), where

195 different species intermingle together. This is caused by two species, namely $S$. warneri and $S$.

196 saccharolyticus, which do not form monophyletic groups and clearly some strains from these species

197 have been mislabelled. Additionally, as an independent strategy, we also carried out an ANI analysis to

198 calculate the relatedness of the strains to the type strains included in this study (see Figure 2). This

199 analysis shows that in terms of their taxonomy most of the genomes have been properly labelled. For

200 instance, all the strains designated as $S$. lugdunensis clustered with the type strain, $S$. lugdunensis NCTC

201 12217, with identity percentages well above 95\% and thus could be assigned to this species (Chun, Oren

202 et al. 2018). The same applies to $78.26 \%$ of the other species; see for example S. gallinarum or $S$.

203 simulans where genomes labelled as belonging to each of these species clustered tightly (again above the

$20495 \%$ ) with the type strains. On the other hand, the mis-classification previously noted in the phylogeny is

205 also evident in this analysis, as the strains of both $S$. warneri and $S$. saccharolyticus do not cluster all

206 together in each case and their identity percentages are well below 95\% (see green labels in Figure 2). We

207 then carried out a GO enrichment analysis to have an idea of the overrepresented functions of the 177 
208 SGFs (see Supplementary Table 4), as expected many of the enriched biological processes and molecular

209 functions have to do with housekeeping functions; the top 2 GO molecular functions were ATP binding

210 and GTP binding, whereas the top 2 Biological processes were DNA repair and fatty acid biosynthesis

211 (see Supplementary Table 4 for more details). Taken together, these results demonstrate that the 177

212 SGFs seem to be real OGs and that most of the genomes have a proper affiliation regarding their

213 taxonomy.

214

215 Ranking the orthologous groups.

216 Then we analysed which of the 177 SGFs, from now on OGs, could be the best markers to infer the

217 evolutionary relationships among the species. For that end, we established how similar each one of the

218 single gene trees (from the 177 OGs) was compared to the PSTT; this was done employing the Robinson

219 and Foulds (RF) distance between the single gene trees and the PSTT - this distance gives the number of

220 bipartitions that are not shared by the two trees under consideration. We also used $\pi$, a common measure

221 of genetic diversity, to establish the amount of genetic variation for each OG. Figure 3 gives the

222 percentage of similarity of the 177 OG trees to the PSTT and the nucleotide diversity for each of the 177

223 OGs; this figure shows that no single OG yielded the same topology as the PSTT. Furthermore, we also

224 noted that every single OG has its own topology - not shared by any other OG - as none of all the

225 pairwise comparisons of the OGs trees gave a RF equal to 0 (a RF distance of 0 implies that the two

226 topologies in comparison are the same, see Supplementary Figure 2). Figure 3 also shows that 167 OGs

227 (94\%) have nucleotide diversity values higher than 0.15 , which make them good candidates for

228 phylogenetic markers. Of note, for two species ( $S$. aureus and S. epidermidis) we also computed the intra-

229 species diversity and it seems that these OGs even at this level have a good amount of genetic diversity

230 (see Supplementary Figure 3). We found that the top 26 OGs were similar to the PSTT, as all of them

231 have RF distances below 214, indicating that they are more than $60 \%$ identical to the PSTT. Notably

232 these 26 OGs present good values of nucleotide diversity (all but two showing values higher than 0.2

233 nucleotide changes per nucleotide site and the average for the 26 being 0.26 nucleotide changes per 
234 nucleotide site), which is very convenient for their use as to phylogenetic markers. Table 1 provides

235 details about these OGs such as RF distance PSTT, nucleotide diversity, function and the ID in the $S$.

236 aureus TW20 genome. Remarkably, when we concatenated these 26 OGs and constructed a phylogeny,

237 this tree showed a topology pretty similar to the PSTT (see Figure 4, panel A), the RF distance was 74

238 being $86.1 \%$ identical to the PSTT. Furthermore, using only the best 7 OGs (the bold candidates from

239 Table 1), we got a similar result - that is the concatenated ML phylogeny of these 7 OGs is almost $80 \%$

240 identical to the PSTT (see panel B, Figure 4). Here it is worth mentioning that although these two

241 phylogenies (Figure 4) are not 100\% identical to the PSTT, the two phylogenies recovered almost all the

242 species as monophyletic groups with very good bootstrap values ( $>80 \%)$, see blue dots in both

243 phylogenies - the only exceptions being the miss-classifications noted also in the PSTT and ANI analysis.

244 In considering this part, although no single gene tree of the 177 OGs yielded the PSTT, just using the best

24526 (and even just the top 7) OGs we were able to recover a topology pretty similar to the PSTT.

246

247 The inadequacy of usual markers and the soundness of our strategy

248 Then, we wanted to know how a set of commonly used markers for inferring the phylogenetic

249 relationships within this genus compared to our list. We chose the genes dnaJ, tuf, sodA, rpoB and hsp60

250 as these are the set of genes most used in previous studies (Kwok and Chow 2003, Ghebremedhin, Layer

251 et al. 2008). Most of them had clear issues to be considered suitable markers given the criteria we

252 employed to define our genuine OGs. Notably two of them, $t u f$ and $r p o B$, did have signals for

253 recombination as shown by their Neighbour nets and the PhiTest (see Supplementary Figure 1) and their

254 nucleotide diversity was very low (below 0.15 , see Figure 3 orange dots). On the other hand, sodA and

255 hsp60 were not single gene families, as they did have more than one gene per genome in some species.

256 Among these usual markers, only dnaJ seems to be a good candidate in as much as it is a SGF, did not

257 have signals of recombination and has a nucleotide diversity value above 0.2 (see Figure 3 ). The

258 shortcoming of most of these genes is not totally unexpected, as these genes were not selected based on 
259 phylogenetic criteria; however, it is clear that most of these genes do not seem to be a good option to infer 260 the history of the species.

261

262 Finally, to prove the validity of our phylogenomic approach, in the data set here employed we included 263 the genome sequences of four bacterial isolates collected by us from cattle ticks and phenotypically 264 classified as members of the genus Staphylococcus (see Supplementary Table 2) - these isolates were 265 sequenced using an Illumina MiSeq platform (see methods). Initially these isolates were classified using

266 the 16S rRNA gene sequence (see Supplementary Table 2); however, in 2 of these cases, namely INIFAP 267 009-16 and INIFAP 002-15, the initial classification was incorrect. For instance, INIFAP 009-16 was 268 classified as S. succinus by its 16S rRNA gene sequence but the PSTT placed this isolate with S. xylosus. 269 In the case of INIFAP 002-15, whereas PSTT assigned it to S. succinus, the starting classification via the $27016 \mathrm{~S} r R N A$ gene was S. xylosus. Importantly, in the concatenate alignments using our 26 OGs (Table 1) 271 the isolates were properly classified (see panel A, Figure 4). Furthermore, the ML phylogeny based on the 272 concatenated alignment of the top 7 OGs also recovers the true affiliation of these isolates (see panel B, 273 Figure 4). To sum up, our genuine OGs are a much better option than the usual markers to establish the 274 phylogeny of the species and even just a few of these (the top 7) are able to adequately carry out 275 taxonomy classification of the newly sequenced bacterial isolates.

276

277 DISCUSSION

278 The main goal of our study was to establish the best markers for species identification in the genus

279 Staphylococcus. To try to be as comprehensive as possible, we used a data set with 46 species and a total 280 of 269 genomes from this genus that broadly represents most if not all the niches cover by this genus.

281 Here we identified 177 OGs and ranked them according to their potential as phylogenetic markers;

282 clearly, this set of markers should be useful not only for ecological and evolutionary studies but also for 283 clinical and biotechnological purposes. In addition, our phylogenomic approach allowed us to identify

284 two species ( $S$. warneri and S. saccharolyticus) in which mislabelling of deposited genomes has occurred. 
285 This issue of misclassification is not that rare, as genome sequences submitted in public databases many

286 times do not undergo a proper inspection in terms of microbial taxonomy. However, the combination of

287 these phylogenomic approaches (genome-based phylogeny and ANI analysis) can be very useful to

288 pinpoint those cases of misclassification.

289

290 We want to emphasise that our study has two major contributions: one is the list of adequate markers for

291 inferring the phylogeny of the species within the genus Staphylococcus and the other is the phylogenomic

292 strategy that we used to identify such markers. Regarding the first major contribution, given that the

293 average gene content of the species here analysed is 2413 , our 177 OGs represent barely the $7 \%$ of the

294 genome of these species. However, we need to emphasise here that, for merely practical reasons, we

295 focused on orthologous genes with one-to-one relationships and, very likely, there are many more

296 orthologous genes within these species that did not pass our criteria given that they are not SGF. Despite

297 the fact that all these OGs are potential good markers, in as much as their histories do not deviate

298 significantly from the history of the species, they do differ among them in the amount of phylogenetic

299 signal they each present. Importantly, no single OG was able to exactly represent the PSTT and no two

300 OGs yielded the same topology; clearly, we found a cloud of topologies. This is in agreement with a

301 previous study that found that only one of the several hundreds of OGs reflected the species tree (Castillo-

302 Ramirez and Gonzalez 2008), although this focused on different evolutionary scales and different

303 bacteria. It is worth mentioning, that the top 26 OGs have good values of nucleotide diversity (all but two

304 have values higher than 0.21), and are localized in different parts of the chromosome and present different

305 functions. We think these 26 OGs represent a really good set of markers for inferring the PSTT; actually

306 concatenating the best 7 it was possible to get a very good approximation of the PSTT. Here, we want to

307 highlight that using the phylogeny of the top 7 OGs all but the 2 species with issues of mis-classification

308 were recovered as monophyletic groups and thus it seems that just using these 7 markers species

309 assignation can be carried out. Clearly, this set of markers could be very useful for underfunded

310 laboratories working with Staphylococcus isolates. Although from a genotyping perspective, ideally one 
311 would want to use whole-genome sequencing for species identification, many laboratories in the world

312 (specially in developing countries) still cannot afford the sequencing of tens to hundreds of isolates. Thus,

313 this short list of markers should be extremely useful for those who only can sequence a few loci, which is

314 often the case for clinical, environmental and evolutionary biology microbiologists in developing

315 countries. Clearly, the markers highlighted in this study are a much better option that the commonly used

316 markers, as the latter seem to exhibit obvious flaws (more than one copy per genome, signals of

317 recombination, low nucleotide diversity values) for inferring the history of the species. Our strategy, and

318 in turn the list of genes found, proved to be factually sound, even when using just a few markers for the

319 taxonomic assignment of newly sequenced bacterial isolates.

320

321 Maybe more important than the first major contribution is the fact that our phylogenomic approach will

322 allow the identification of adequate markers in many different genera - not only from bacteria but also

323 from archaea. This is very important, as gene families showing only orthologous relationships very likely

324 do not extend all over the tree of life - or just for a few gene families. Along these lines, it is very likely

325 that many of the markers found here will not work for other genera (i.e. they will not show one-to-one

326 orthologous relationships) as the homologous genes within those genera might have been affected by

327 horizontal gene transfer or duplication and differential loss, or some other molecular event that prevent

328 the history of the gene to reflect the speciation events. We want to highlight that the biology of the

329 species under consideration could have a very important effect on the number of orthologous genes found.

330 For instance, highly recombinogenic species, such as Neisseria gonorrhoeae (Ezewudo, Joseph et al.

331 2015) and Acinetobacter baumannii (Grana-Miraglia, Lozano et al. 2017), would have fewer orthologous

332 genes than more clonal species. Nonetheless, the strategy employed by us should work even in these

333 species, although the number of potential orthologous genes should be much less.

334

335 CONCLUSIONS 
336 In summary, here we devised and applied a phylogenomic approach that allowed us to define the most

337 suitable markers for inferring the species phylogeny within the genus Staphylococcus. We acknowledge

338 that the effectiveness of these markers for other bacterial genera remains open to investigation. However,

339 we are confident that the phylogenomic approach here implemented can be employed to identify the most

340 suitable markers for other genera. On a broader level, this study has very practical implications, as it

341 provides a general framework for mapping out the best markers to construct the phylogeny of the taxa

342 considered not only at the genus level but also at other taxonomic levels.

343

344

345

346

347

348

349

350

351

352

353

354

355

356

357

358

359

360

361

362

363

364

365

366

\section{Acknowledgments}

We are extremely grateful to the editor and the reviewers, as their suggestions have drastically improved our manuscript.

\section{REFERENCES}

Bankevich, A., S. Nurk, D. Antipov, A. A. Gurevich, M. Dvorkin, A. S. Kulikov, V. M. Lesin, S. I. Nikolenko, S. Pham and A. D. Prjibelski (2012). "SPAdes: a new genome assembly algorithm and its applications to single-cell sequencing." \ournal of computational biology 19(5): 455477.

Becker, N. S., G. Margos, H. Blum, S. Krebs, A. Graf, R. S. Lane, S. Castillo-Ramírez, A. Sing and V. Fingerle (2016). "Recurrent evolution of host and vector association in bacteria of the Borrelia burgdorferi sensu lato species complex." BMC genomics 17(1): 734.

Borowiec, M. L. (2016). "AMAS: a fast tool for alignment manipulation and computing of summary statistics." PeerJ 4: e1660.

Bradley, R. K., A. Roberts, M. Smoot, S. Juvekar, J. Do, C. Dewey, I. Holmes and L. Pachter (2009). "Fast statistical alignment." PLoS computational biology 5(5): e1000392.

Bruen, T. C., H. Philippe and D. Bryant (2006). "A simple and robust statistical test for detecting the presence of recombination." Genetics 172(4): 2665-2681.

Castillo-Ramirez, S. and V. Gonzalez (2008). "Factors affecting the concordance between orthologous gene trees and species tree in bacteria." BMC Evol Biol 8: 300. 
367 Challagundla, L., J. Reyes, I. Rafiqullah, D. O. Sordelli, G. Echaniz-Aviles, M. E. Velazquez-

368 Meza, S. Castillo-Ramírez, N. Fittipaldi, M. Feldgarden and S. Chapman (2018).

369 "Phylogenomic Classification and the Evolution of Clonal Complex 5 Methicillin-Resistant

370 Staphylococcus aureus in the Western Hemisphere." Frontiers in Microbiology 9: 1901.

371 Chun, J., A. Oren, A. Ventosa, H. Christensen, D. R. Arahal, M. S. da Costa, A. P. Rooney, H. Yi,

372 X. W. Xu, S. De Meyer and M. E. Trujillo (2018). "Proposed minimal standards for the use of

373 genome data for the taxonomy of prokaryotes." Int I Syst Evol Microbiol 68(1): 461-466.

374 Cox, M. P., D. A. Peterson and P. J. Biggs (2010). "SolexaQA: At-a-glance quality assessment

375 of Illumina second-generation sequencing data." BMC bioinformatics 11(1): 485.

376 Ezewudo, M. N., S. J. Joseph, S. Castillo-Ramirez, D. Dean, C. Del Rio, X. Didelot, J.-A. Dillon, R.

377 F. Selden, W. M. Shafer and R. S. Turingan (2015). "Population structure of Neisseria

378 gonorrhoeae based on whole genome data and its relationship with antibiotic resistance."

379 Peer 3: e806.

380 Fitch, W. M. (2000). "Homology a personal view on some of the problems." Trends Genet

381 16(5): 227-231.

382 Frisch, M., S. Castillo-Ramirez, R. Petit, M. Farley, S. Ray, V. Albrecht, B. Limbago, J.

383 Hernandez, I. See and S. Satola (2018). "Invasive methicillin-resistant Staphylococcus

384 aureus USA500 strains from the US Emerging Infections Program constitute three

385 geographically distinct lineages." mSphere 3(3): e00571-00517.

386 Gabaldón, T. and E. V. Koonin (2013). "Functional and evolutionary implications of gene

387 orthology." Nature Reviews Genetics 14(5): 360.

388 Ghebremedhin, B., F. Layer, W. König and B. König (2008). "Genetic classification and

389 distinguishing of Staphylococcus species based on different partial gap, 16S rRNA, hsp60,

390 rpoB, sodA, and tuf gene sequences." Journal of clinical microbiology 46(3): 1019-1025.

391 Grana-Miraglia, L., L. F. Lozano, C. Velazquez, P. Volkow-Fernandez, A. Perez-Oseguera, M.

392 A. Cevallos and S. Castillo-Ramirez (2017). "Rapid Gene Turnover as a Significant Source of

393 Genetic Variation in a Recently Seeded Population of a Healthcare-Associated Pathogen."

394 Front Microbiol 8: 1817.

395 Huson, D. H. and D. Bryant (2005). "Application of phylogenetic networks in evolutionary

396 studies." Molecular biology and evolution 23(2): 254-267.

397 Joseph, S. J., D. Cox, B. Wolff, S. S. Morrison, N. A. Kozak-Muiznieks, M. Frace, X. Didelot, S.

398 Castillo-Ramirez, J. Winchell and T. D. Read (2016). "Dynamics of genome change among

399 Legionella species." Scientific reports 6: 33442.

400 Joseph, S. J., H. Marti, X. Didelot, S. Castillo-Ramirez, T. D. Read and D. Dean (2015).

401 "Chlamydiaceae genomics reveals interspecies admixture and the recent evolution of

402 Chlamydia abortus infecting lower mammalian species and humans." Genome biology and 403 evolution 7(11): 3070-3084.

404 Kwok, A. Y. and A. W. Chow (2003). "Phylogenetic study of Staphylococcus and

405 Macrococcus species based on partial hsp60 gene sequences." International journal of

406 systematic and evolutionary microbiology 53(1): 87-92.

407 Lamers, R. P., G. Muthukrishnan, T. A. Castoe, S. Tafur, A. M. Cole and C. L. Parkinson (2012).

408 "Phylogenetic relationships among Staphylococcus species and refinement of cluster

409 groups based on multilocus data." BMC evolutionary biology 12(1): 171.

410 López-Leal, G., M. L. Tabche, S. Castillo-Ramírez, A. Mendoza-Vargas, M. A. Ramírez-Romero

411 and G. Dávila (2014). "RNA-Seq analysis of the multipartite genome of Rhizobium etli CE3 
412 shows different replicon contributions under heat and saline shock." BMC genomics 15(1): 413770.

414 Parks, D. H., M. Imelfort, C. T. Skennerton, P. Hugenholtz and G. W. Tyson (2015). "CheckM:

415 assessing the quality of microbial genomes recovered from isolates, single cells, and 416 metagenomes." Genome research: gr. 186072.186114.

417 Richter, M. and R. Rosselló-Móra (2009). "Shifting the genomic gold standard for the 418 prokaryotic species definition." Proceedings of the National Academy of Sciences 106(45): 419 19126-19131.

420 Sasaki, T., S. Tsubakishita, Y. Tanaka, A. Sakusabe, M. Ohtsuka, S. Hirotaki, T. Kawakami, T. 421 Fukata and K. Hiramatsu (2010). "Multiplex-PCR method for species identification of 422 coagulase-positive staphylococci." Џournal of clinical microbiology 48(3): 765-769.

423 Stamatakis, A. (2014). "RAxML version 8: a tool for phylogenetic analysis and post-analysis 424 of large phylogenies." Bioinformatics 30(9): 1312-1313.

425 Zerbino, D. and E. Birney (2008). "Velvet: algorithms for de novo short read assembly using 426 de Bruijn graphs." Genome research: gr. 074492.074107. 


\section{Figure 1 (on next page)}

Maximum likelihood phylogenetic tree based on the super alignment of the orthologous groups.

Maximum likelihood phylogenetic tree based on the super alignment of the 177 nonrecombinant SGFs. Strains of the same species are labelled with the same colour and black stars indicate type strain for some species. Green and violet rectangles highlight strains with conflicting clade assignment, S. warneri and S. saccharolyticus respectively. Bootstrap values above 80 are shown in orange dots. The scale bar shows the number substitutions per site. The code for the strain identifiers is in Supplementary Table 1. 


\section{Figure 2 (on next page)}

Heat map of the Average Nucleotide Identity (ANI) analysis of the 269 strains from Staphylococcus spp.

The reddish cells show identity percentages above $95 \%$ implying that the strains belong to the same species, whereas non-reddish colors denote identity percentages below 95\% (see ANI key). The top and left side rows on the heat map show the species assignation (see species key). The dendograms across the side and top show the clustering of the strains; strain identifiers as in Figure 1. Color of the strain identifiers (bottom row) is as follows: red gives the type strains, whereas blue shows the strains with issues of mis-classification. This analysis indicates 44 clearly discernibleStaphylococcus spp. 


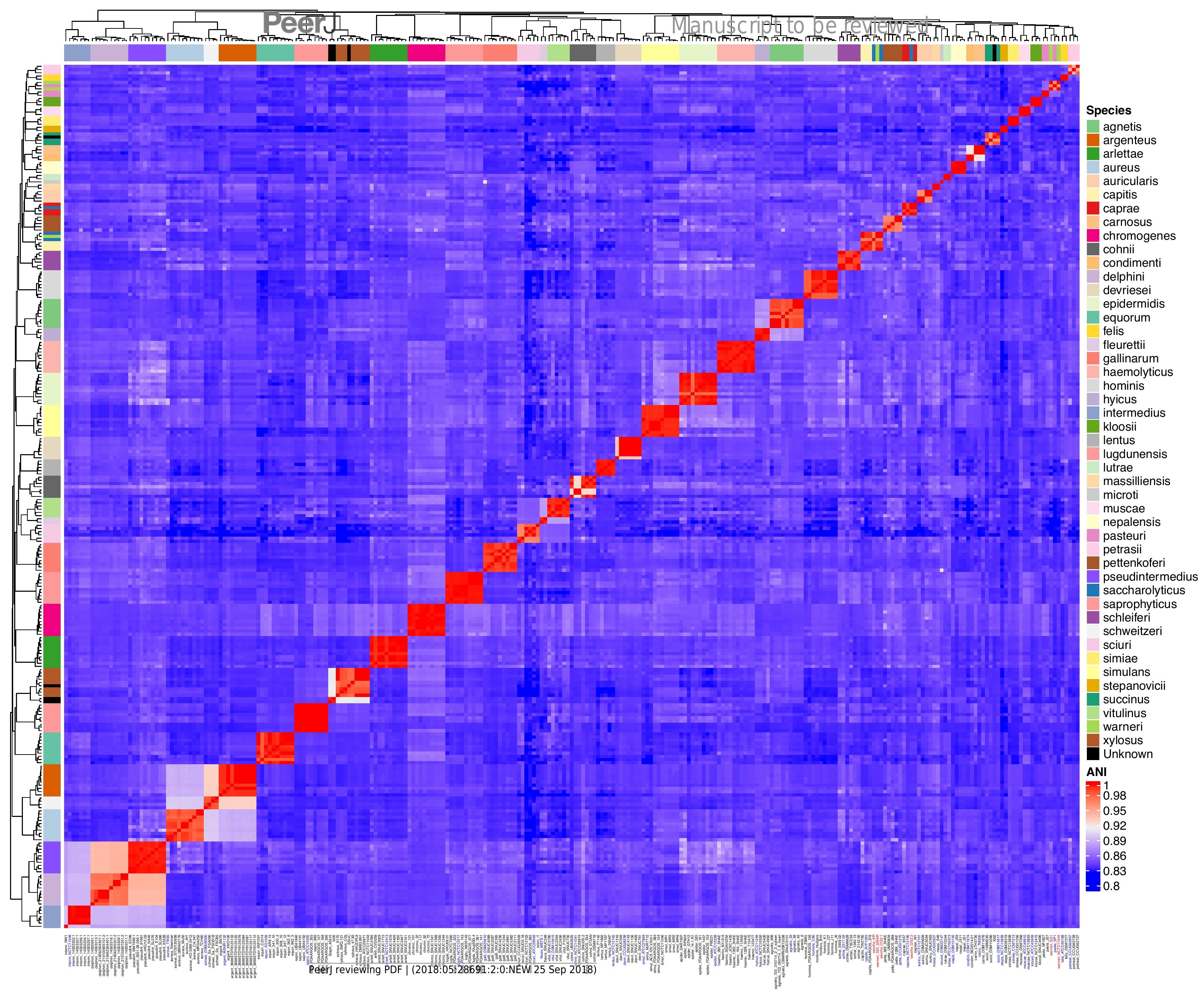




\section{Figure 3}

Similarity to the species tree and nucleotide diversity

Percentage of similarity between gene trees and the PSTT and the nucleotide diversity for each of the 177 SGF (green dots). The most similar gene tree topologies correspond to genes with high levels of nucleotide diversity. The commonly used marker genes dnaJ, rpoB and tuf are highlighted (orange dots), they all show low to moderate nucleotide diversity values and none of them show a similarity percentage to the PSTT above $60 \%$.

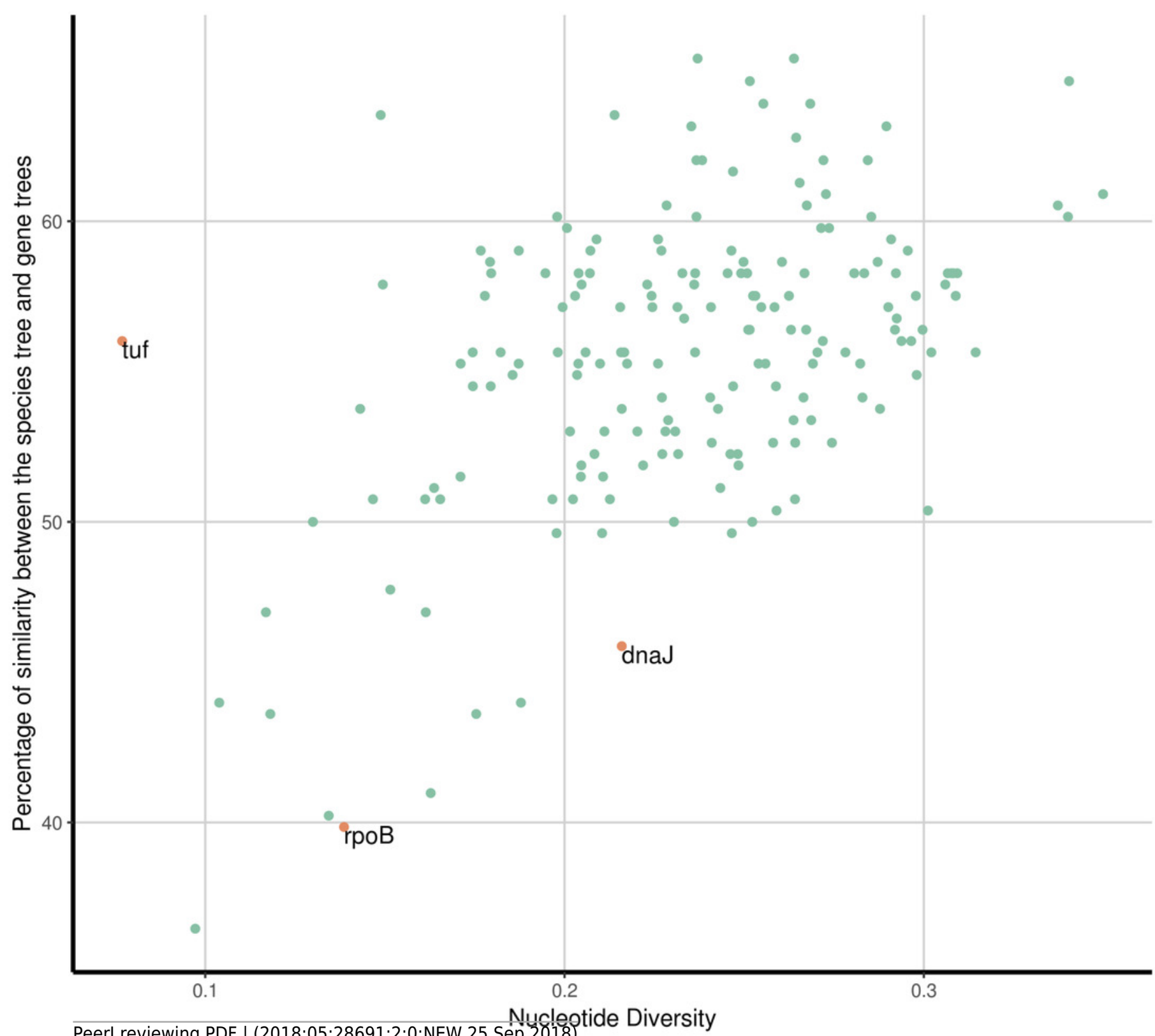


Figure 4

Phylogenies of the top orthologous groups

Phylogenetic trees based on the concatenated alignments of the 26 orthologous groups listed in Table $1(A)$ and on the concatenated alignments of the top seven orthologous groups (B). The tree in panel A has a percentage of similarity with the PSTT of $86 \%$ and the tree in panel B of $79 \%$. Bootstrap values above 80 are shown in blue dots. Scale bar shows the number of substitutions per site.
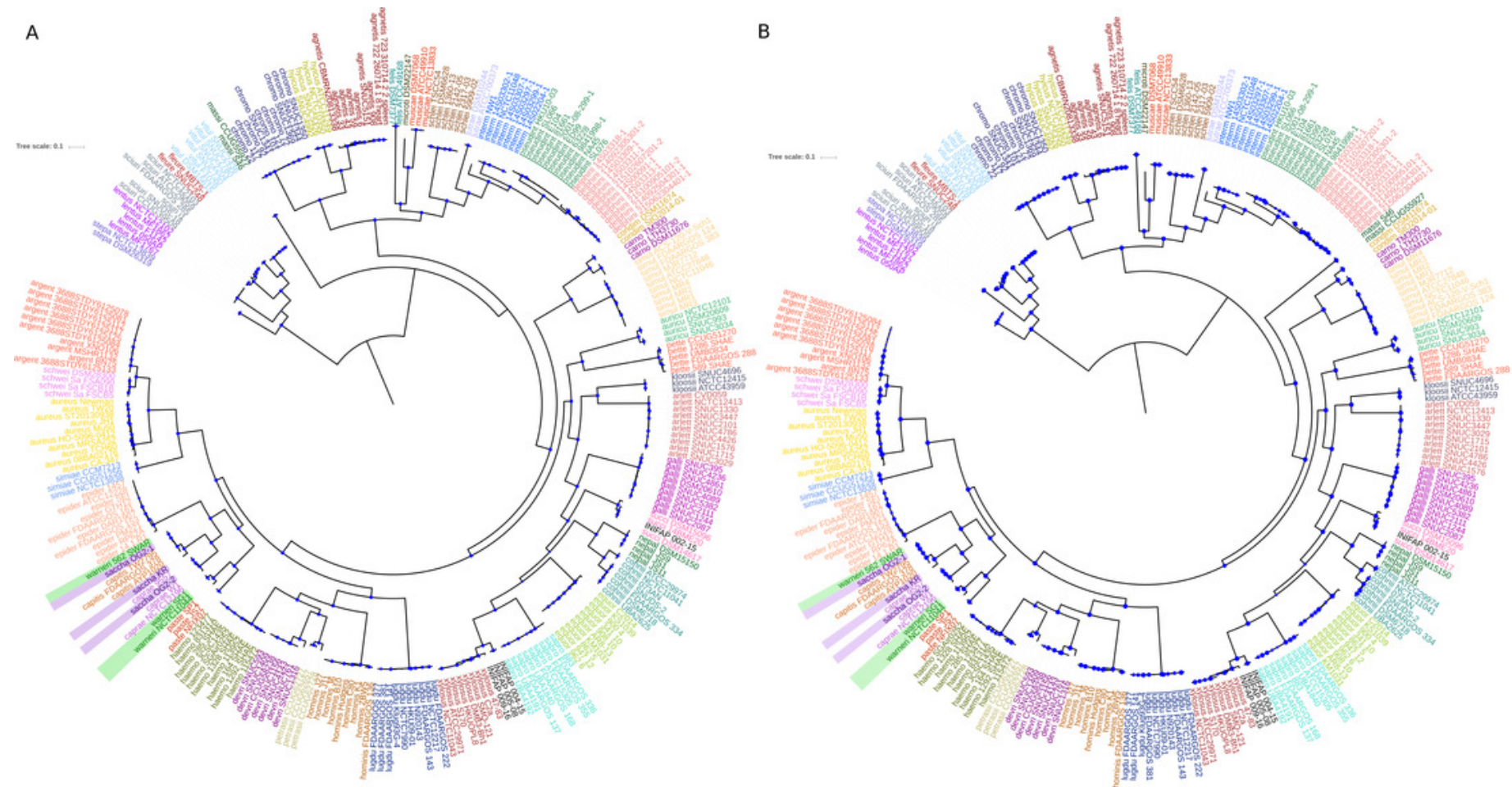


\section{Table $\mathbf{1}$ (on next page)}

Top 26 genuine orthologous genes

These are the most adequate markers for inferring the species phylogeny. * Gene names were taken from the UniProt (www.uniprot.org) database when possible, otherwise the proteins were blasted against NCBI protein database (www.ncbi.nlm.nih.gov) and a wellannotated Staphylococcus species was used for annotation. 


\begin{tabular}{|c|c|c|c|c|c|}
\hline Description* & $\begin{array}{l}\text { NCBI Reference } \\
\text { Sequence }\end{array}$ & GC content & Nucleotide Diversity & $\begin{array}{l}\text { Proportion of Variable } \\
\text { sites }\end{array}$ & $\begin{array}{c}\text { Similarity with } \\
\text { Species Tree } \\
\text { (Robinson-Fould } \\
\text { Distance) }\end{array}$ \\
\hline $\begin{array}{l}\text { DNA mismatch } \\
\text { repair protein } \\
\text { (MutS) }\end{array}$ & WP_000073352.1 & 0.342 & 0.2370335 & 0.412 & $65.4135(184)$ \\
\hline $\begin{array}{l}\text { ATP-dependent } \\
\text { RecD-like DNA } \\
\text { helicase (recD2) }\end{array}$ & WP_001283311.1 & 0.345 & 0.2638535 & 0.588 & $65.4135(184)$ \\
\hline $\begin{array}{c}\text { Cytosol } \\
\text { aminopeptidase } \\
(\text { pepA })\end{array}$ & WP_001009697.1 & 0.361 & 0.3404309 & 0.760 & 64.6617 (188) \\
\hline $\begin{array}{c}\text { putative ABC } \\
\text { transporter ATP- } \\
\text { binding protein } \\
\text { (YheS) }\end{array}$ & WP_000602071.1 & 0.339 & 0.2515735 & 0.688 & $64.6617(188)$ \\
\hline $\begin{array}{l}\text { Phenylalanine- } \\
\text { tRNA ligase beta } \\
\text { subunit ( } p h e T \text { ) }\end{array}$ & WP_000908982.1 & 0.366 & 0.2683997 & 0.696 & 63.9098 (192) \\
\hline $\begin{array}{l}\text { Penicillin-binding } \\
\text { protein H (pbp3) }\end{array}$ & WP_000919772 & 0.340 & 0.2553319 & 0.623 & $63.9098(192)$ \\
\hline DNA-directed & & & & & \\
\hline $\begin{array}{l}\text { RNA polymerase } \\
\text { subunit beta' } \\
\text { (rpoC) }\end{array}$ & CBI48492.1 & 0.381 & 0.1488365 & 0.442 & $63.5338(194)$ \\
\hline $\begin{array}{l}\text { Pyruvate kinase } \\
\qquad(p y k)\end{array}$ & WP_001232648.1 & 0.361 & 0.2139191 & 0.584 & 63.5338 (194) \\
\hline DNA polymerase & & & & & \\
\hline $\begin{array}{l}\text { III subunit alpha } \\
\qquad(D n a E)\end{array}$ & WP_000226911.1 & 0.336 & 0.2895834 & 0.737 & $63.1579(196)$ \\
\hline $\begin{array}{c}\text { UDP-N- } \\
\text { acetylmuramate-L- } \\
\text { alanine ligase } \\
(\text { murC })\end{array}$ & WP_000150163.1 & 0.335 & 0.2352931 & 0.622 & 63.1579 (196) \\
\hline fibronectin-binding & & & & & \\
\hline $\begin{array}{c}\text { domain-containing } \\
\text { protein }(Y / O A)\end{array}$ & WP_000312763.1 & 0.338 & 0.2644582 & 0.667 & $62.782(198)$ \\
\hline
\end{tabular}


UDP-N-

acetylmuramoyl-L-

alanyl-D-

WP_000340119.1 0.374

0.2366963

0.638

$62.0301(202)$

glutamate-L-lysine

ligase (murE)

Homoserine

dehydrogenase

WP_000735864.1 0.344

0.2720552

0.579

$62.0301(202)$

(dhoM)

Acetyl-coenzyme A

carboxylase

carboxyl

WP_000883645.1 0.357

0.2383029

0.584

$62.0301(202)$

transferase subunit

alpha $(\operatorname{acc} A)$

Molybdopterin

molybdenumtransf WP_000259718.1 0.386

0.2844009

0.679

$62.0301(202)$

erase $($ MoeA)

S-

adenosylmethionin

e:tRNA WP_001019171.1 0.357

0.2468871

0.620

$61.6541(204)$

ribosyltransferase-

isomerase (queA)

ATP-dependent

DNA helicase

WP_000983677.1 0.345

0.2654353

0.659

$61.2782(206)$

(RecQ)

hypothetical

protein (YibE/F- $\quad$ WP_001794550.1 0.363

0.3498756

0.723

$60.9023(208)$

like)

DNA polymerase

III subunit tau

WP_001109047.1 0.368

0.2727606

0.645

$60.9023(208)$

$($ dnaX)

Ribonuclease $\mathrm{R}$

(rnr)

WP_001050064.1 0.367

0.2284088

0.626

$60.5263(210)$

Ktr system

potassium uptake WP_000021864.1 0.342

0.2674265

0.646

$60.5263(210)$

protein B $(k t r B)$

Dihydrolipoyl

dehydrogenase WP_001291535.1 0.353

0.3373007

0.759

$60.5263(210)$

(pdhD)

3-oxoacyl-[acyl- WP_001100524.1 0.377

0.2367141

0.601

$60.1504(212)$ 


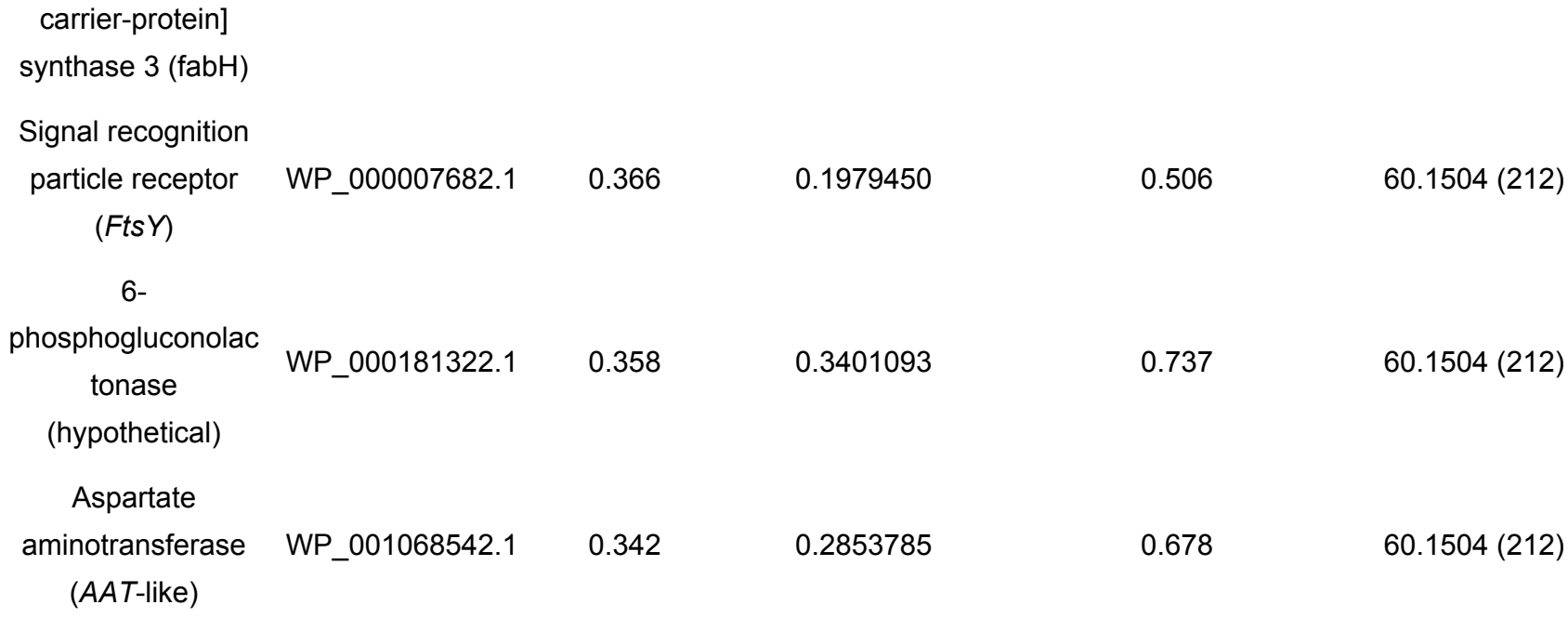

